\title{
A Surrogate Model for Interference Prevention in the Limaçon- to-Limaçon Machines
}

\author{
Ibrahim A. Sultan \\ School of Science and Engineering \\ The University of Ballarat \\ PO Box 663 \\ Ballarat 3353 VICTORIA, Australia \\ Email: i.sultan@ballarat.edu.au
}

\begin{abstract}
The work presented in this paper is concerned with the limaçon-to-limaçon fluid processing machine, which is a rotary positive displacement device used for either energy production or utilisation. Limaçon-to-limaçon machines have their housings and rotors both manufactured of limaçon profiles and exhibit rotor-housing interference if their rotors are not carefully designed. However, the analysis presented in published literature for the interference problem yields a nonlinear iterative procedure which would be useful if used to calculate the clearance values for a given design with given dimensions. However, such an iterative procedure would not be suitable if employed in an extensive optimisation exercise in which hundreds of different designs with different combinations of dimensions are searched for the optimum solution. For these applications, it would be more efficient if the iterative inference model is substituted by a fitted equation (or a surrogate model) which possesses high accuracy in the region of interest. The procedure undertaken to obtain the surrogate model is described in this work, and the results of the accuracy tests are presented.
\end{abstract}

\section{Introduction}

Surrogate models are common to various areas of scientific endeavour where the exact mathematical form of a relationship underlying a certain process is not readily obtainable. In this case, empirical methods are usually employed together with suitable regression techniques to work out a substitution functional expression relating the various parameters controlling the process. Bates et al (2000) represent a good 
example of this type of work as they propose a fitted model to emulate the effects of some engine parameters on its performance indices. Gilsinn et al (2002), combine the cubic splines with the least squares fitting method in an elegant procedure to describe the machining errors in a turning process. Various methods used to obtain fitted mathematical models are introduced by Montgomery and Reltz (2000), who also propose an optimisation technique based on the concept of two-level factorial. Indeed, the process of obtaining a fitted model is essentially an optimisation exercise, where the error between the estimated system output and its measured value is to be minimised. Some researchers such as Jeang et al (2002) employ the method of Monte Carlo simulation to obtain data sets of input parameters needed for the analysis. This simulation technique is explained in simple terms by Hanson and Hemez (2001), who also point out a few examples of its application in engineering experimentations. However, Soh et al (2003) argue that this method is time consuming and does not reveal the system input-output relationship in a straightforward manner. Instead they propose an alternative simulation technique, intended to reduce the number of observations, which features the use of both the mean and the standard deviation in the analysis.

Invaluable reviews of the statistical implications of fitted models are found in the papers by Carley et al (2004) and Robinson et al (2004). In these two papers special attention is paid to the method of response surface, which systematically leads to a fitted optimised model developed around a region of interest. Lee et al (2002) employ the method to describe the stiffness of joints on vehicle structures using a fitted model. In fact the majority of published work describes models that have been fitted using gradient-based techniques. However, Fan et al (2000) argue that these techniques always lead to local, rather than global, convergence. As such, they advocate the use of Genetic Algorithms which are more likely to exhibit global convergence, despite their slow disposition. Roux et al (1998) worked around this convergence problem by developing their response surface model about a small region of interest in the design space. Bray and Rhinehart (2000) head in a different direction as they advocate the use of mechanistic models. These models are usually based on a physical description of the process at hand. Even though the power-law distribution models which are based on the linear fit of the log-log scale are common tools in statistically-based analysis, Goldstein et al (2004) conduct numerical 
experiments to conclude that these models provide biased estimates for the power exponent. Instead they propose the use of the maximum likelihood estimation which is more robust.

The work presented in this paper is concerned with the limaçon-to-limaçon fluid processing machine, which is a rotary positive displacement device used for either energy production (e.g. air motors, expanders and engines) or utilisation (e.g. compressors and pumps). Limaçon-to-limaçon machines have their housings and rotors both manufactured of limaçon profiles, as suggested by Sultan (2006). A few mechanical linkages have been proposed and patented by designers to drive the rotors of limaçon machines. Sultan (2005) describes some of these linkages and comments on their mechanical characteristics. Sultan (2006) points out that the rotor-housing interference is a phenomenon which might have hindered progress in the area of limaçon machinery; and consequently presents a mathematical insight into the phenomenon and proposes design solutions. However, the analysis presented by Sultan (2006) shows that the geometric model which describes the interference problem yields a nonlinear iterative procedure which would be useful if used to calculate clearance values for a given design with given dimensions. However, such an iterative procedure would not be suitable if employed in an extensive optimisation exercise in which hundreds of different designs with many different combinations of dimensions are searched for the optimum solution. For these applications, it would be more efficient if the iterative inference model is substituted by a non-iterative surrogate model which possesses high accuracy in the region of interest. This concept motivated the work presented in this paper. In the next section the geometric equations of the limaçon-to-limaçon machines will be presented.

\section{Geometric aspects of a limaçon-to-limaçon machine}

Figure (1) depicts a limaçon-to-limaçon machine when its rotor has performed a rotational displacement, $\theta$. The housing limaçon is developed about a circle referred to as the base circle. The vertical axis (i.e. the Y-axis in the figure) of this circle coincides with the line about which the housing limaçon is symmetric. Moreover, the lowest point, o, on the circumference of the base circle is referred to as the limaçon 
pole. When the rotor is at the horizontal position, its centre point, $\mathrm{m}$, coincides with the pole of the housing limaçon. During rotations, the rotor axis (also referred to as the limaçon chord) stays kinematically attached to this pole, and the centre point of this axis always falls on the circumference of the base circle. By virtue of the drive linkages, the rotor performs sliding and rotational motions about the housing pole whilst its apices always touch the housing to provide sealing action. As Figure (1) shows, at any angular position, $\theta$, the distance, $\mathrm{s}$, through which the rotor has slid over the housing pole is equal to the distance from the rotor centre point to this pole. This distance can be expressed as follows;

$s=2 r \sin \theta$

where $r$ is the radius of the housing base circle.

Based on the above description, it is worth noting that the radial distance measured on the chord from the housing pole to point $\mathrm{p} 1$ is given as $s+L$, and the $\mathrm{X}$ - and $\mathrm{Y}$ coordinates of the housing profile, $\mathrm{x}_{\mathrm{h}}$ and $\mathrm{y}_{\mathrm{h}}$ respectively, can be described with respect to a stationary XY-Cartesian frame situated at the limaçon pole, as shown in Figure (1), as follows;

$x_{h}=r \sin 2 \theta+L \cos \theta$

and

$y_{h}=r-r \cos 2 \theta+L \sin \theta$

where $\mathrm{L}$ is half the chord length. $\theta \in[0,2 \pi]$ is the angle rotated by the rotor-axis (or the chord) as its apex, p1, touches the housing at the point ( $\left.\mathrm{x}_{\mathrm{h}}, \mathrm{y}_{\mathrm{h}}\right)$. In fact (2) and (3) are the general Cartesian equations of the limaçon curve.

Sultan (2005) refers to the ratio $\mathrm{r} / \mathrm{L}$ as the aspect ratio of the limaçon. Costa et al (1999) present an interesting discussion on the effect of the aspect ratio on the shape of the limaçon curve. For a limaçon curve to be suitable for fluid processing, it has to be free of dimples and looping, which can be achieved by limiting the value of the aspect ratio to 0.25 .

It is worth reminding the reader here not to confuse the limaçon curve with another member of the epitrochoidal family, namely the curve employed for the housing of the well-known Wankel engine; where the $L \cos \theta$ and $L \sin \theta$ terms in equations (2) 
and (3) are, respectively, replaced by $L \cos (2 \theta / 3)$ and $L \sin (2 \theta / 3)$. Whilst the rotor of a Wankel-type machine is three-lobed, the rotor of a limaçon-to- limaçon machine has a lenticular profile and is machined out of two identical limaçon curves, mirrorimaged about its centre-line. The rotor lower limaçon can be described with respect to a coordinate system, $\mathrm{XrYr}$, situated at the rotor centre point as shown in Figure (1). This coordinate system is rigidly attached to the rotor and moves with it. The $\mathrm{Xr}$ - and Yr- coordinates, $\mathrm{x}_{\mathrm{rl}}$ and $\mathrm{y}_{\mathrm{rl}}$ respectively, of any point on the lower half of the rotor profile are given as follow;

$x_{r l}=r_{r} \sin 2 \phi+(L-C) \cos \phi$

and

$y_{r l}=r_{r}-r_{r} \cos 2 \phi+(L-C) \sin \phi$

where $r_{r}$ is the radius of the base circle about which the rotor lower limaçon is developed, and $\phi \in[\pi, 2 \pi]$ is an angle measured in the CCW direction from the $\mathrm{Xr}$ axis to a point on the rotor profile. Figure (1) indicates the base circles of the rotor lower and upper halves. In this work, the housing and rotor base circles are taken to have equal radii. This is a common case with limaçon machinery, particularly those which are intended to handle gaseous fluids. Smooth running conditions are made possible if the rotor half-width, L, is shortened by a distance $\mathrm{C}$, as indicated in equations (4) and (5). These two coordinate equations can also be used to describe the upper half of the rotor profile, except the $y_{\mathrm{rl}}$ value has to be multiplied by -1 .

\section{The rotor-housing clearance}

Figure (2) depicts the instant where the rotor has been displaced by an angle $\theta$, as measured from the $\mathrm{X}$-axis. At this instant, it is required to calculate the radial distance, $\Delta$, between any point, such as $\mathrm{p} 3$, on the rotor profile and its corresponding point, $\mathrm{p} 4$, on the housing limaçon. Both $\mathrm{p} 3$ and $\mathrm{p} 4$ fall on the same radial line $\mathrm{R}_{\mathrm{h} \varepsilon}$, which describes the housing limaçon with respect to its pole, o. The instantaneous angle of $R_{h \varepsilon}$ as measured from the $X$-axis is $\theta+\varepsilon$. The angle, $\beta$, of the radial line, $R_{r}$, which defines $\mathrm{p} 3$ with respect to the rotor limaçon pole, $\mathrm{m}$, is measured from the $\mathrm{Xr}$ axis. $\mathrm{R}_{\mathrm{r} \beta}$ can be expressed as follows;

$R_{r \beta}=2 r_{r} \sin (\beta+\pi)+(L-C)$ 
where $\beta \in[0, \pi]$, and $\pi$ is added to ensure compliance with the condition, $\phi \in[\pi, 2 \pi]$ above. Likewise, $\mathrm{R}_{\mathrm{h} \varepsilon}$ can be written as;

$R_{h \varepsilon}=2 r \sin (\theta+\varepsilon)+L$

Projecting the different sides of the triangle, omp3, on the $\mathrm{Y}_{\mathrm{r}^{-}}$and $\mathrm{X}_{\mathrm{r}}$-axes, the following relationships can be obtained;

$R_{r \beta} \sin \beta=h \sin \varepsilon$

and

$R_{r \beta} \cos \beta+s=h \cos \varepsilon$

where $\mathrm{s}$ is the rotor sliding distance as given in equation (1). The distance, h, may be calculated, from (1), (8) and (9), as follows;

$h=\sqrt{R_{r \beta}^{2}+4 r^{2} \sin (\theta)^{2}+4 r R_{r \beta} \sin \theta \cos \beta}$

Now, $\Delta$ can be worked out from Figure (2) as follows;

$\Delta=R_{h \varepsilon}-h$

which may be re-written in the following form;

$\Delta=2 r \sin \theta \cos \varepsilon+2 r \cos \theta \sin \varepsilon+L-h$

The variables $h$ and $\varepsilon$ may now be eliminated from (12) using equations (8), (9) and (10). The resulting relationship is a non-linear function of $\theta, \beta, \mathrm{C}$ and the other limaçon parameters. This relationship is given as follows;

$\Delta=\left(\frac{-2 r \sin (\theta-\beta)-R_{r \beta}}{\sqrt{R_{r \beta}^{2}+4 r^{2} \sin (\theta)^{2}+4 r R_{r \beta} \sin \theta \cos \beta}}\right) R_{r \beta}+L$

In practice, it is preferable to employ the aspect ratio, $b \in(0,0.25]$, in the design equations (rather than the base circle radius, $r$ ) because it has a well defined numerical bounds unlike the absolute value of $r$. As such, the aspect ratio can be employed to express $\Delta$ in the following form;

$\Delta=\left(\frac{-2 b \sin (\theta-\beta)-\frac{R_{r \beta}}{L}}{\sqrt{\frac{R_{r \beta}^{2}}{L^{2}}+4 b^{2} \sin (\theta)^{2}+4 b \frac{R_{r \beta}}{L} \sin \theta \cos \beta}}\right) R_{r \beta}+L$ 
If the value of the radial clearance, $\mathrm{C}$, is not set large enough, $\Delta$ will assume negative values over ranges of the rotational displacement, $\theta$, and the radial line angle, $\beta$. These ranges depend on the limaçon dimensions. Naturally, negative values for $\Delta$ indicate the occurrence of the rotor-housing interference. Also, a value of $\mathrm{C}$ larger than necessary would end up reflecting negatively on the thermodynamic performance of the limaçon-to-limaçon device. Hence, the proper value of $\mathrm{C}$, just needed to ensure that the rotor-housing clearance will not fall beyond a given value, has to be calculated and employed for the design. This is described in the next section.

\section{Interference prevention}

To prevent rotor-housing interference in limaçon machinery, iterative mathematical procedure is carried out to calculate the radial clearance, $\mathrm{C}$, that should be machined off the rotor face in order that the minimum distance separating the rotor from the housing during rotation, would not fall below a certain desired value, $\Delta_{\text {all }}$. This mathematical procedure involves solving the following three nonlinear equation simultaneously;

$\Delta-\Delta_{\text {all }}=0$

where $\Delta$ is as indicated in equation (14),

$\frac{\partial \Delta}{\partial \theta}=0$

and

$$
\frac{\partial \Delta}{\partial \beta}=0
$$

Equations (16) and (17) ensure that the minimum allowable clearance, $\Delta_{\text {all }}$, is assigned at the angular situation where the rotor-housing clearance is at its lowest value. As such the employed iterative procedure produces, on top of the value of radial clearance, $\mathrm{C}$, the angular position of the minimum rotor-housing clearance in terms of the two angles, $\theta$ and $\beta$. 
In fact, the success of the convergence process to a global minimum does depend on initial guess as so does the number of iterations involved. This makes the iterative procedure unsuitable if the clearance analysis was used as part of a large simulation package intended to search for a proper machine dimensions for a specific application and then conduct predictive performance analysis under hundreds of different clearance and operating conditions. For this sort of simulation it would be necessary to replace the iterative procedure with a closed form equation to produce the required radial clearance needed to ensure that the rotor housing clearance would not fall being a desired value. In this work, a closed form curve fitting model is proposed and tested for accuracy and validity. The details of the proposed model are presented in the next section.

\section{Numerical experiments}

Inspecting the model present in equations (15), (16) and (17), it should be straight forward to conclude that the radial clearance, $\mathrm{C}$, is dependent on the values of the three control parameters, $\mathrm{b}, \mathrm{L}$ and $\Delta_{\text {all }}$, i.e. $C=f\left(b, L, \Delta_{\text {all }}\right)$. The model proposed in this paper follows a simplified design practice by setting $r_{r}=r=b L$. It is necessary to conduct a set of numerical experiments to investigate how these independent variables affect the value of the clearance, C. Such an investigation would lead to a fitted model capable of representing the mathematical behaviour of the system with an acceptable degree of fidelity. The numerical experiments conducted in this work employed input vectors given as follows;

$\mathbf{L}=\left[L_{j}\right]$,

$\Delta_{\text {all }}=\left[\Delta_{\text {all } j}\right]$

and

$\mathbf{b}=\left[b_{j}\right]$

where $j=0,1,2, . ., 10$ and the input numerical domains are designed as follows;

$L_{j}=20+20 j$,

$\Delta_{\text {all }}=0+0.5 j$

and

$b_{j}=0.04+0.02 j$ 
where $L_{j}$ and $\Delta_{\text {allj }}$ are given in $\mathrm{mm}$, and the various ranges are expected to be commonly employed in the field of fluid processing machinery. Greater values for L may result in inertia and vibration problems, let alone negative impact on the device volumetric and thermal efficiencies. Also values of $\Delta_{\text {all }}$ greater than the assumed range is likely to impair the characteristics of sealing process. Moreover, values of $b$ greater than 0.25 will result in an uneven limaçon curve, not suitable for fluid processing.

Figure (3) depicts the results of a numerical experiment where the value of rotor halfwidth, L, has been varied as per (19) and the value of C was obtained, iteratively, for four sets of $\mathrm{b}$, and $\Delta_{\text {all }}:\left\{b_{2}, \Delta_{\text {all }}\right\},\left\{b_{2}, \Delta_{\text {all } 10}\right\},\left\{b_{10}, \Delta_{\text {all } 0}\right\}$ and $\left\{b_{10}, \Delta_{\text {all } 10}\right\}$. The figure reveals that the C-L relationship is a linear one; this linearity was confirmed with a line fitting exercise which produced correlation coefficients of 1 for various C-L lines. Moreover, the slope of these lines chiefly depends on the value of the aspect ratio, b. Figure (4), where b has been assigned the values given in (19), confirms this dependency and suggests that $\Delta_{\text {all }}$ has a very small effect on the slope of the C-L lines.

To gain an insight into the manner in which $\Delta_{\text {all }}$ affects the value of the system response $\mathrm{C}$, a numerical experiment has been conducted where $\Delta_{\text {all }}$ was made to vary and $\mathrm{C}$ was obtained for four sets of $\mathrm{L}$ and $\mathrm{b}:\left\{b_{2}, L_{0}\right\},\left\{b_{2}, L_{10}\right\},\left\{b_{10}, L_{0}\right\}$ and $\left\{b_{10}, L_{10}\right\}$. The results of this experiment are shown in Figure (5). The figure clearly reveals that also $\mathrm{C}$ and $\Delta_{\text {all }}$ are related by straight line equations. In fact a straight-line fitting exercise, conducted to confirm the apparent linearity in Figure (5), produced correlation coefficients of 1 .

To the naked eye, the straight lines in Figure (5) possess the slope of unity. However it would be necessary to look more closely at the aspects of this slope. To do so, Figure (6), where $b$ has been assigned the values given in (19), was drawn to reveal the weak dependence of the slope of the C- $\Delta_{\text {all }}$ line on the values of both $\mathrm{b}$ and L. In fact the dependence of the slope on $\mathrm{L}$ is even much weaker than the dependence on $\mathrm{b}$. The insight acquired through the set of numerical experiments presented in this section will be used to propose the response surface model in the next section. 


\section{The fitted model}

The discussion presented in the above section calls for the following model to be proposed;

$C_{\text {fitted }}=\Delta_{\text {all }} F_{1}+L\left(F_{2}+F_{3} \Delta_{\text {all }}\right)$

where $C_{\text {fitted }}$ is the fitted system response, and $F_{1}, F_{2}$ and $F_{3}$ are functions expressed in terms of the input $b$. Even though a few options were investigated for these functions, the following forms have been found to produce favourable results;

$$
\begin{aligned}
& F_{1}=K_{0}+K_{1} b+K_{2} b^{2}+K_{3} b^{4}+K_{4} b e^{-K_{5} / b}, \\
& F_{2}=K_{6}+K_{7} b+K_{8} b^{2}+K_{9} b^{4}+K_{10} b e^{-K_{11} / b} \\
& \quad \text { and } \\
& F_{3}=K_{12}+K_{13} b+K_{14} b^{2}+K_{15} b^{4}
\end{aligned}
$$

where $K_{0}, K_{1}, K_{2}, \ldots, K_{15}$ are the coefficients that have to be found using a suitable regression technique. The coefficients in $F_{3}$ will have the units of $1 / \mathrm{mm}$, and will represent a small numerical contribution to the C-L slope.

To find the model coefficients, 450 various data sets comprising values for $b, \mathrm{~L}$ and $\Delta_{\text {all }}$, were generated randomly using a Monte Carlo simulator and their corresponding clearance values, $\mathrm{C}$, have been calculated using the iterative procedure based on the nonlinear exact model. A least squares estimation model has therefore been constructed and solved using a Levenberg-Marquardt procedure as given by the following equation;

$$
\boldsymbol{\delta} \mathbf{K}=\left(\mathbf{J}^{T} \mathbf{J}+\lambda \mathbf{I}\right)^{-1} \mathbf{J}^{T} \mathbf{E}
$$

where $\delta \mathrm{K}$ is the necessary correction in the value of the coefficient vector at a certain iteration, $\mathbf{J}$ is the system Jacobian, $\mathbf{I}$ is a $16 \times 16$ identity matrix and $\mathbf{E}$ is a vector comprising the difference between the exact value of the clearance, $\mathrm{C}$, and its corresponding estimated value, $\mathrm{C}_{\text {fitted }}$. $\lambda$ in the above equation is a scalar quantity chosen initially large to ensure numerical stability for the computational procedure, and is then reduced gradually during iterations to enhance the speed of convergence.

The convergence criterion was adopted as follows; 


$$
\|\delta \mathbf{K}\| \leq \varepsilon
$$

where $\varepsilon$ is a small positive number and $\|\cdot\|$ denotes the Euclidean norm.

The regression procedure produced the following values for the model coefficients; $K_{0}=1.001, K_{1}=-0.05, K_{2}=0.719, \mathrm{~K}_{3}=12.036, K_{4}=2.165, K_{5}=0.725$, $K_{6}=8.354 \times 10^{-5}, K_{7}=-5.641 \times 10^{-5}, K_{8}=0.139, K_{9}=8.464, K_{10}=-0.548, K_{11}=0.862$, $K_{12}=-1.141 \times 10^{-5}, K_{13}=4.314 \times 10^{-4}, K_{14}=-3.7471 \times 10^{-3}, K_{15}=0.019$

The procedure undertaken to validate the proposed model is described out in the next section.

\section{Model validation}

To validate the model 150 data sets of $\mathrm{b}, \mathrm{L}$ and $\Delta_{\text {all }}$, have been randomly generated, using a Monte Carlo simulator, and employed in the nonlinear iterative model to obtain the exact value of the required radial clearance, $C$. The same data sets have also been used in equation (20) to obtain the value of $\mathrm{C}_{\text {fitted }}$. The deviation, $E$, in the fitted value of $\mathrm{C}$ was then calculated as follows;

$E=C-C_{\text {fitted }}$

Also the percentage error, $E \%$, has been defined as follows;

$$
E \%=\frac{C-C_{\text {fitted }}}{C} \times 100
$$

A histogram was plotted for the percentage error as shown in Figure (7), and a spectrum was drawn for the absolute deviation in Figure (8).

To inspect the goodness of fit, the formulas given by Carley et al (2004) have been used to calculate the coefficient of multiple determination, $\mathrm{R}^{2}$ and its adjusted value, $\mathrm{R}_{a d j}^{2}$. They have both been found to be equal to 1 . Moreover the prediction error sum of squares (PRESS) was found to be 0.002436, and the mean value of the sum of absolute errors was calculated as $0.001754 \mathrm{~mm}$. This is very small compared to the mean value of the exact response which was found to be $3.513 \mathrm{~mm}$. 
The standard deviation of the exact values of $\mathrm{C}$ was $2.003 \mathrm{~mm}$, the standard deviation of the absolute error was calculated to be $0.00326 \mathrm{~mm}$, and its root mean square is $0.00327 \mathrm{~mm}$. The standard deviation of the percentage errors was found to be $0.09363 \%$. These small error values confirm the validity of the fitted model and its suitability to substitute the lengthy iterative procedure in the region of interest.

Figures (9) and (10) indicate how the fitted-model features linear C-L and C- $\Delta_{\text {all }}$ relationships comparable to these present in the original iterative procedure.

\section{$\underline{\text { Conclusions }}$}

Limaçon-to-limaçon machines have their rotors and housings machined of the limaçon curve. Without proper clearance introduced, interference will take place resulting in rotor-housing friction, impact loading, reduced reliability and deteriorated machine performance. Iterative procedure exists to calculate the amount of clearance needed to be machined off the rotor profile to ensure that interference is prevented. However, this procedure is not suitable for implementation in lengthy optimisation techniques where a simplified surrogate model would be preferable. The work presented here describes the procedure undertaken to find this model and check its accuracy. The proposed model has been found accurate and capable of maintaining the main geometric features of the original model, which proves the validity of the proposed technique and its suitability for the intended application.

\section{$\underline{\text { References }}$}

1. Bray, R.P. and Rhinehart, R.R., "A Method of Mechanistic Model Validation with a Case Study in Plasma Etching," Plasma Chemistry and Plasma Processing, Vol. 21(1), pp. 163-174, 2000.

2. Bates, R.A., Fontana, R., Randazzo, C. Vaccarino, E. and Wynn, H.P., "Empirical Modelling of Diesel Engine Performance for Robust Engineering Design," in Statistics for Engine Optimization, Edited by Edwards, S.P., Grove, D.M. and Wynn, H.P., Professional Engineering Publishing, pp. 163-173, 2000. 
3. Carley, K.M., Kamneva, N.Y. and Reminga, J., "Response Surface Methodology," CASOS Technical Report, CMU-ISRI-04-136, Carnegie Mellon University, 2004.

4. Costa, S. I. R., Grou, M. A. and Figueiredo, V, "Mechanical Curves - A Kinematic Greek Look Through the Computer," Int. J. of Mathematical Education in Science and Technology, 30(3), pp. 459-468, 1999.

5. Draper, N. and Smith, H., “Applied Regression Analysis," Wiley series in probability and mathematical statistics. John Wiley \& Sons, New York, 1981.

6. Fan, H.-Y., Lu, W.-Z. J. and Xu, Z.-B., "An Empirical Comparison of Three Novel Genetic Algorithms,” Engng Computations, Vol. 17(8), pp. 981-1001, 2000.

7. Gilsinn, D.E., Brandy, H.T. and Ling, A., “A Spline Algorithm for Modelling Cutting Errors on Turning Centres,” J. Intelligent Manuf. Vol. 13, pp. 391-401, 2002.

8. Goldstein, M.L., Morris, S.A. and Yen, G.G., "Problems with Fitting to the PowerLaw Distribution,” Eur. Phys. J., B 41, pp. 255-258, 2004.

9. Hemez, F.M. and Hanson, K.M, "A Framework for Assessing Confidence in Computational Predictions," Experimental Techniques, pp. 50-55, July/August 2001.

10.Jeang, A., Chen., T.-K. and Hwan, C.-L., "A Statistical Dimension and Tolerance Design for Mechanical Assembly Under Thermal Impact," Int. J. Adv. Manuf. Technol. Vol. 20, pp. 907-915, 2002.

11.Lee, S.B., Park, J.R. and Yim, H.J., "Numerical Approximation of Vehicle Joint Stiffness by Using Response Surface Method," Int. J. Automotive Technology, Vol. 3(3), pp. 117-122, 2002.

12.Montgomery, D.T. and Reitz, R.D., "Applying Design of Experiments to the Optimization of Heavy-Duty Diesel Engine Operating Parameters," in Statistics for Engine Optimization, Edited by Edwards, S.P., Grove, D.M. and Wynn, H.P., Professional Engineering Publishing, pp. 163-173, 2000. 
13.Robinson, T.J., Borror, C.M. and Myers, R., "Robust Parameter Design: A Review," Qual. Reliab. Engng. Int.,Vol. 20, pp. 81-101, Dec. 2003.

14.Roux, W.J., Stander, N. and Hafika, R.T., "Response Surface Approximations for Structural Optimization,” Int. J. Numer. Meth. Engng. Vol. 42, pp. 517-534, 1998.

15. Soh, Y.C., Lam, Y.C. and Wu, Z., "Efficient Algorithm for Probabilistic Analysis of Complex Function in Engineering Applications,” Engng Computations, Vol. 21(5), pp. 540-559, 2004.

16.Sultan, I.A., "Profiling Rotors for Limaçon-to-Limaçon Compression-Expansion Machines," ASME Transaction, J. Mechanical Design, Vol. 128(4), 2006.

17.Sultan, I.A., "The Limaçon of Pascal: Mechanical Generation and Utilization for Fluid Processing," Proc. IMechE Vol. 219(8) Part C: J. Mechanical Engineering Science, 2005. 


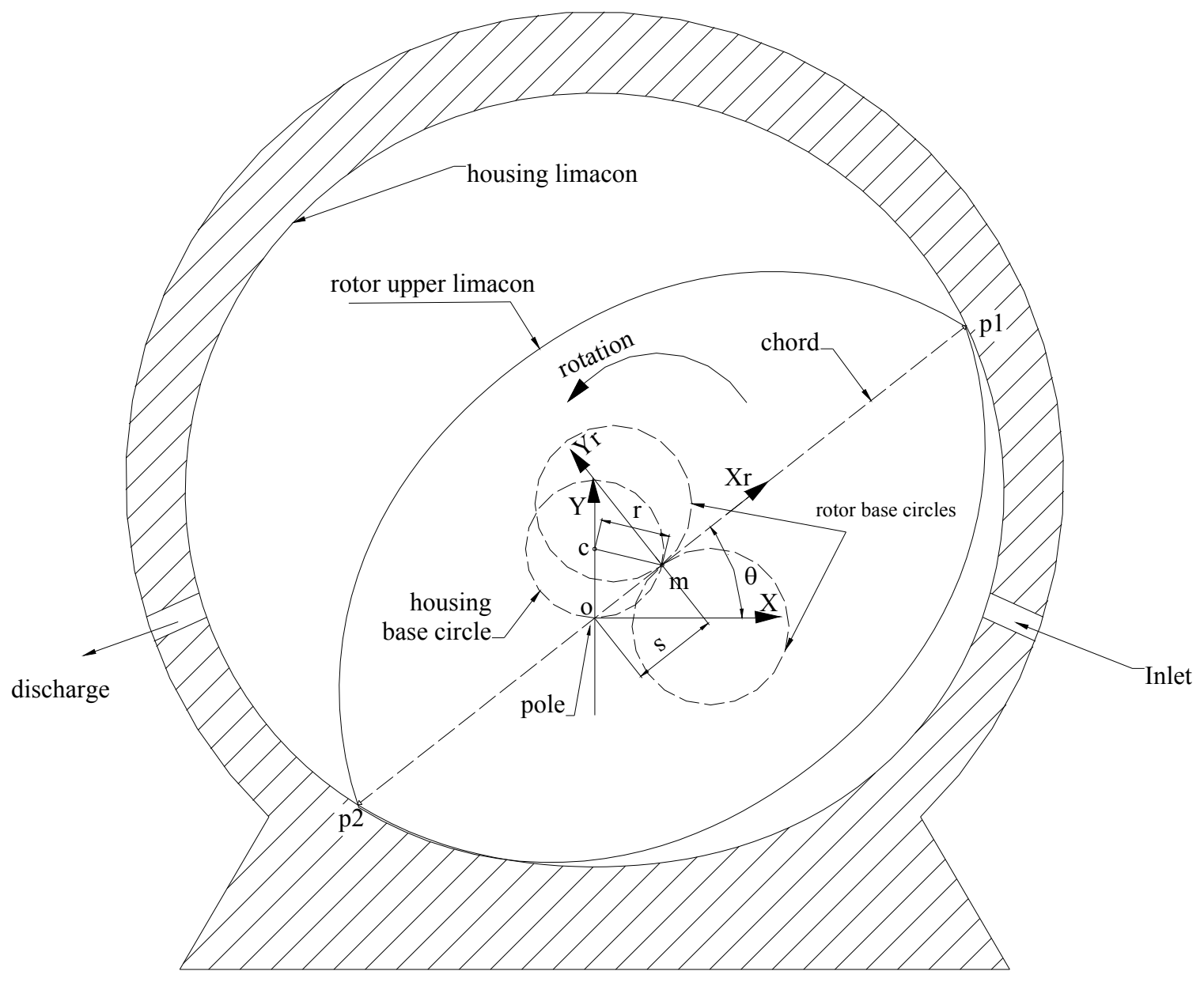

Figure 1. A limaçon-to-limaçon machine. 


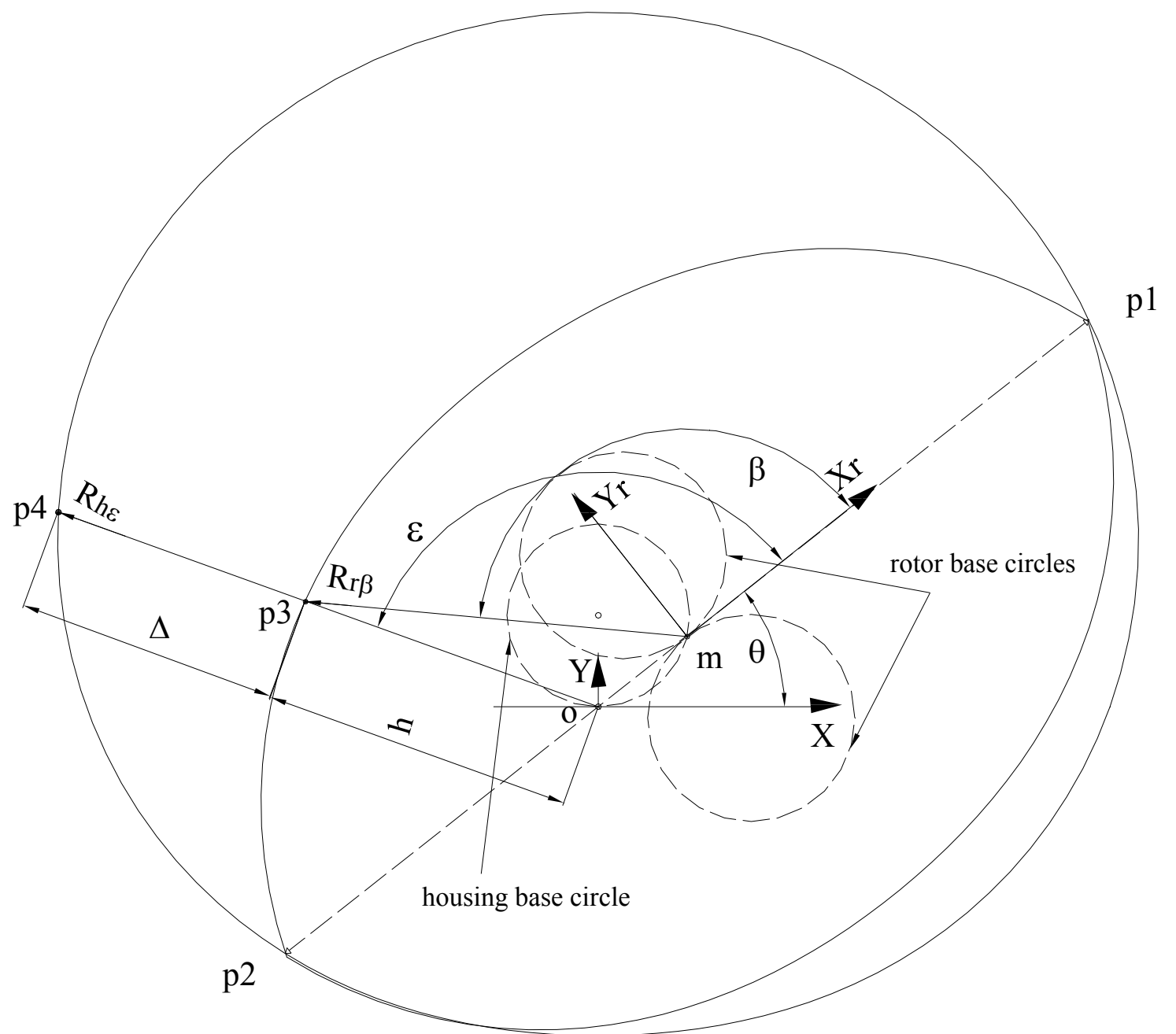

Figure 2. Developing the clearance equations. 


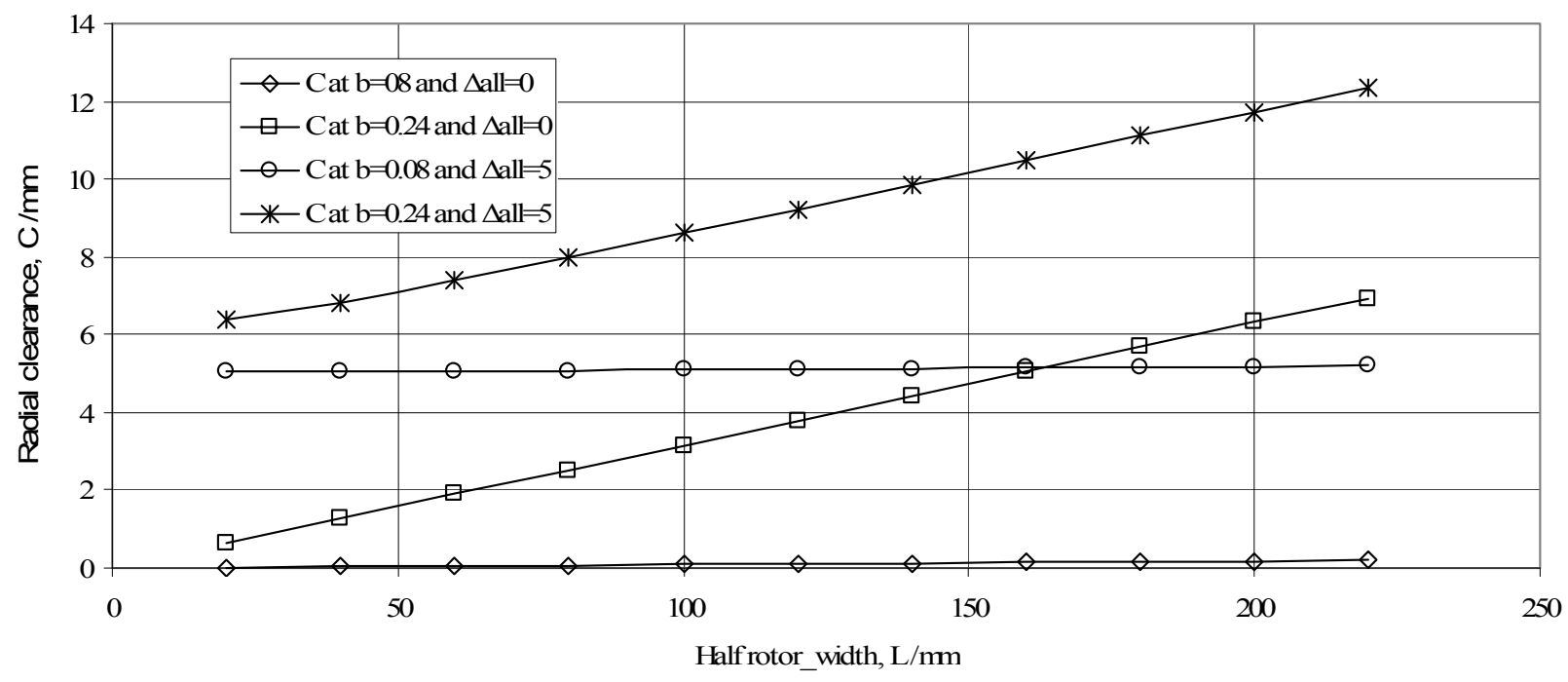

Figure 3. Radial clearance against rotor half-width.

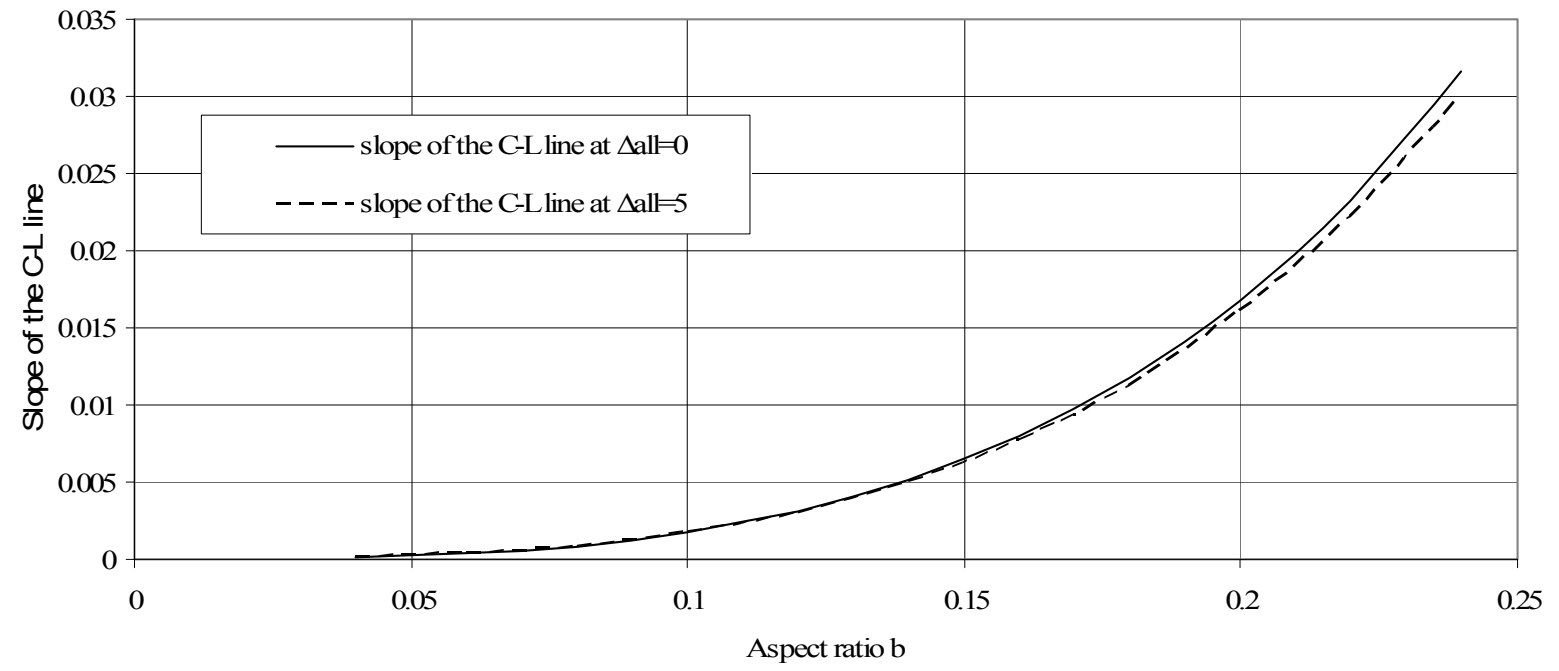

Figure 4. Slopes of the C-L lines and their dependency on the aspect ratio, b. 


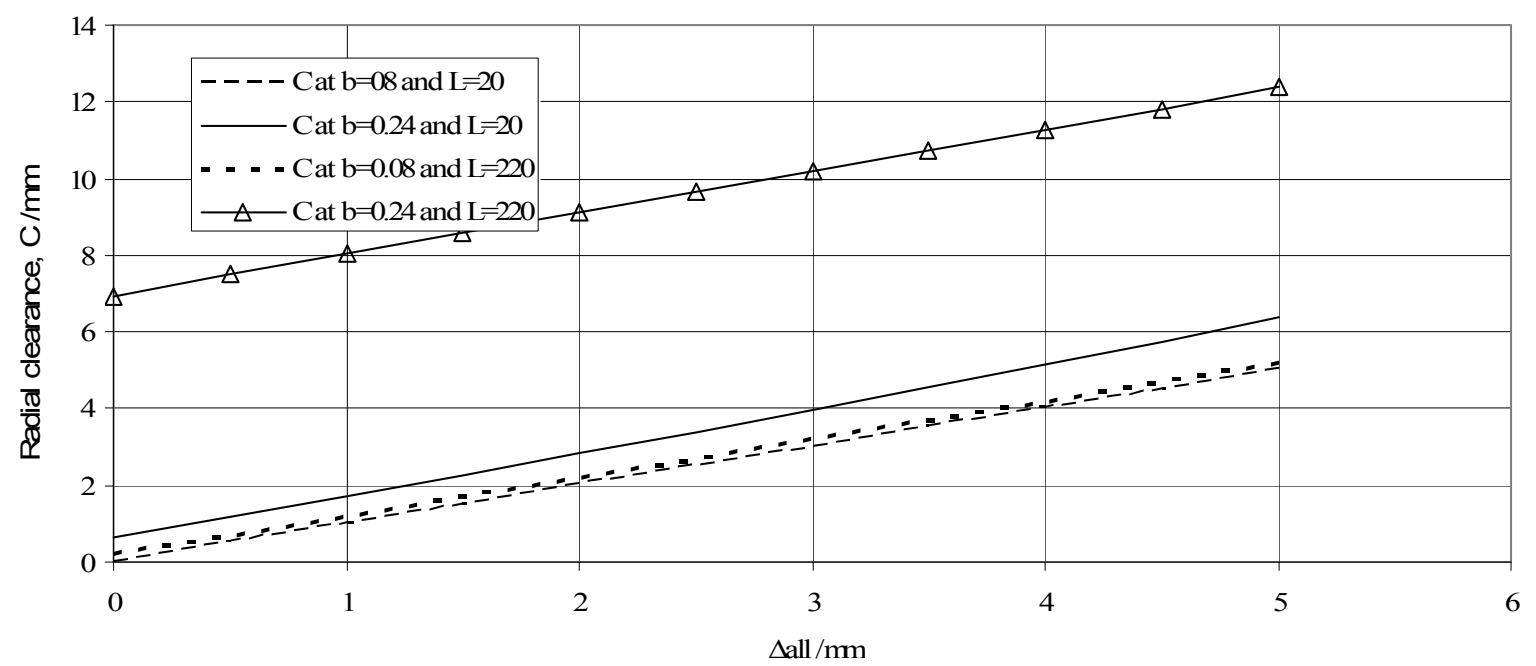

Figure 5. Radial clearance against the allowable minimum clearance.

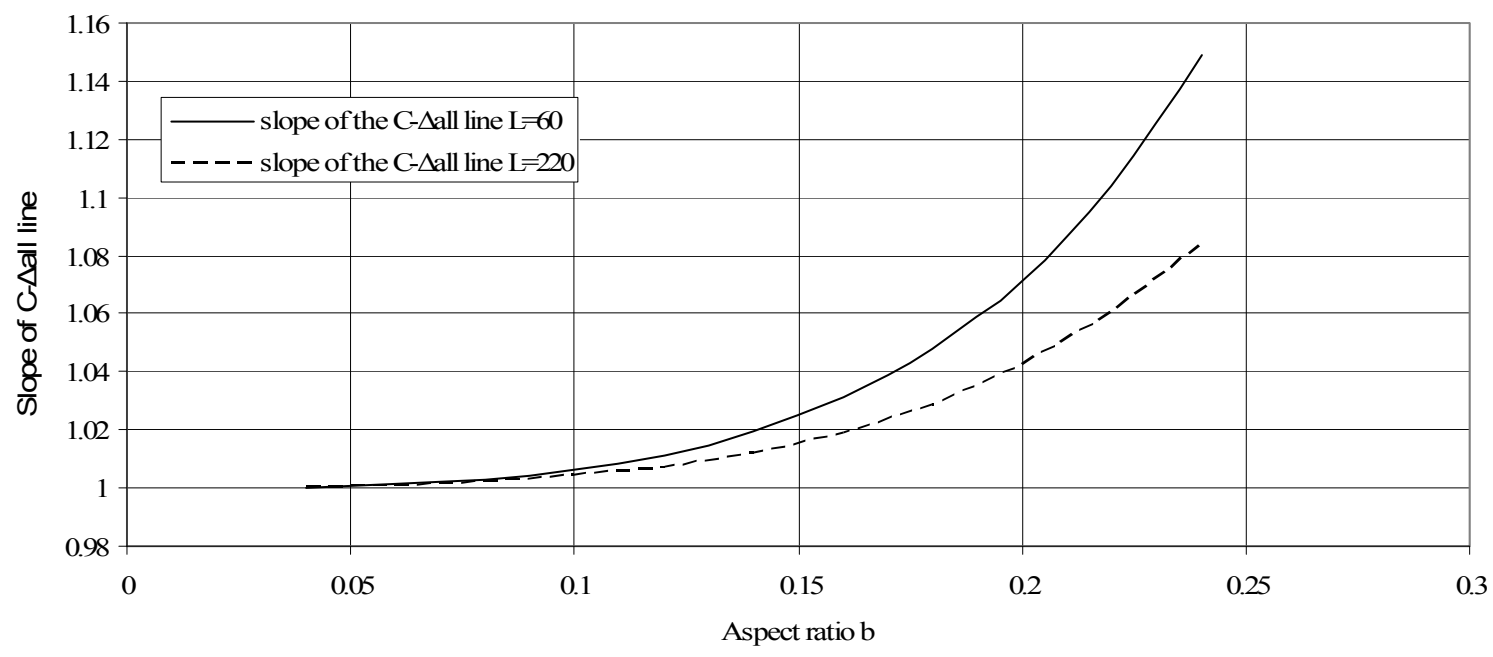

Figure 6. Slopes of the C- $\Delta_{\text {all }}$ lines and their dependency on $\mathrm{b}$ and L. 


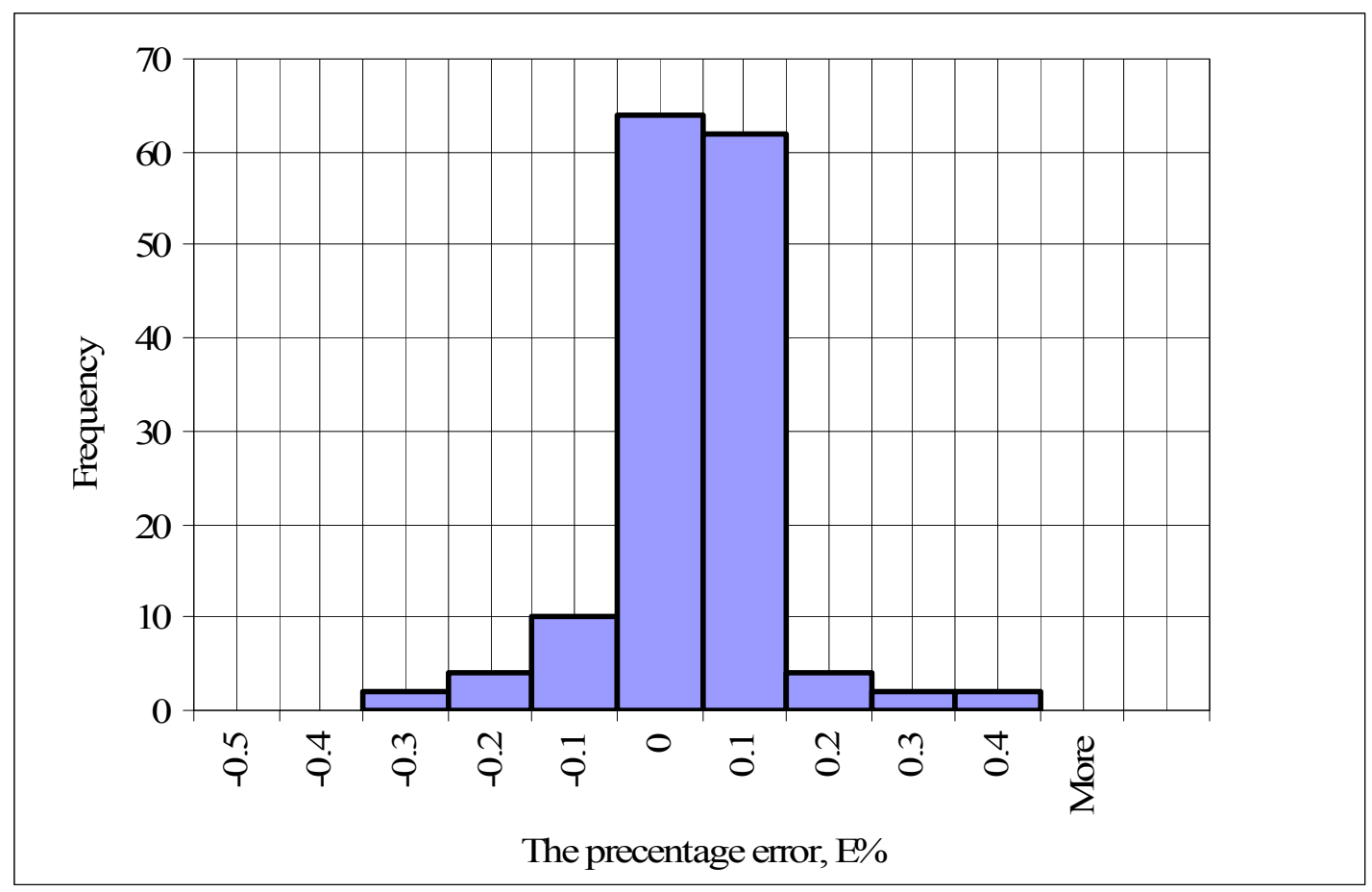

Figure 7. A histogram of the percentage error for randomly generated data sets.

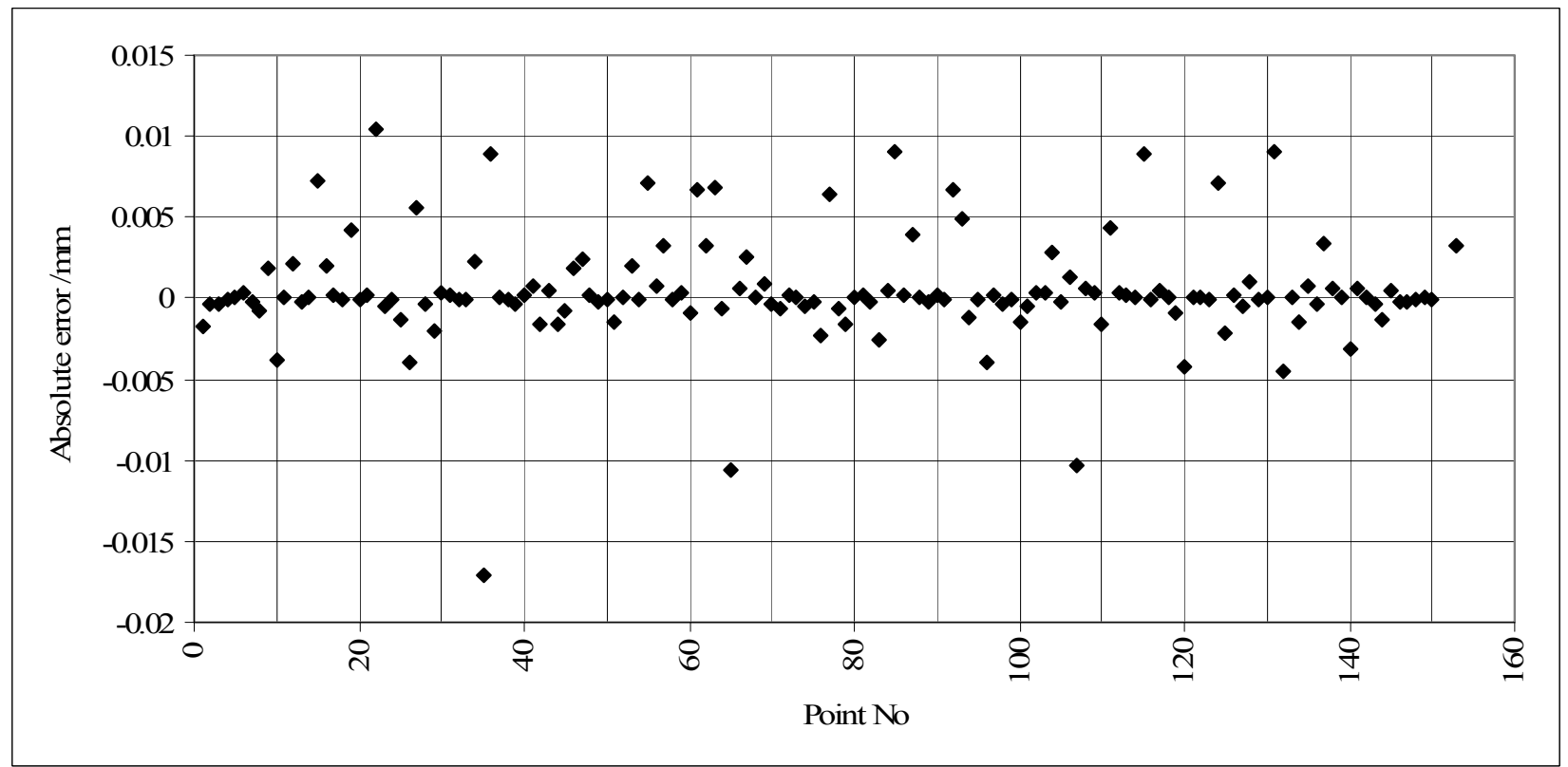

Figure 8. A spectrum of the absolute deviation for randomly generated data sets 


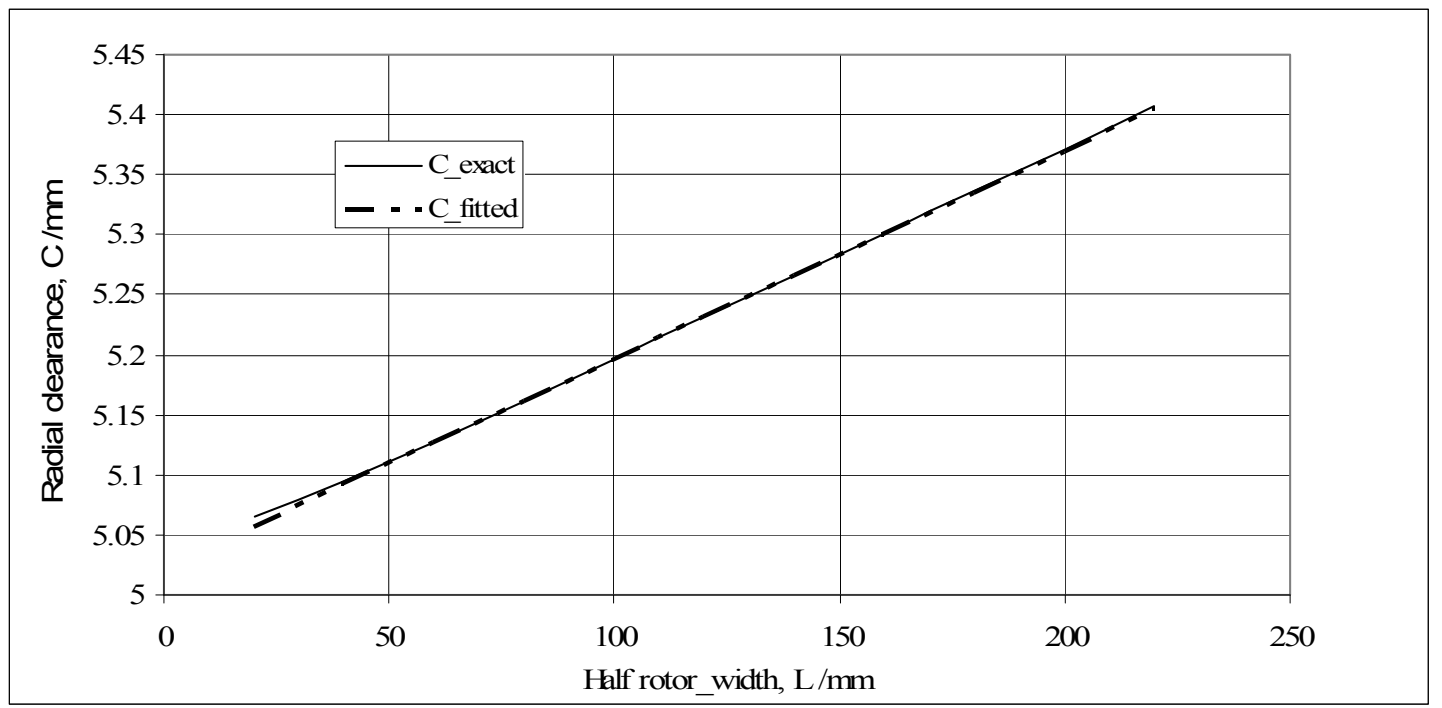

Figure 9. The C-L linearity of the fitted model, for $\mathrm{b}=0.1$ and $\Delta_{\text {all }}=5 \mathrm{~mm}$.

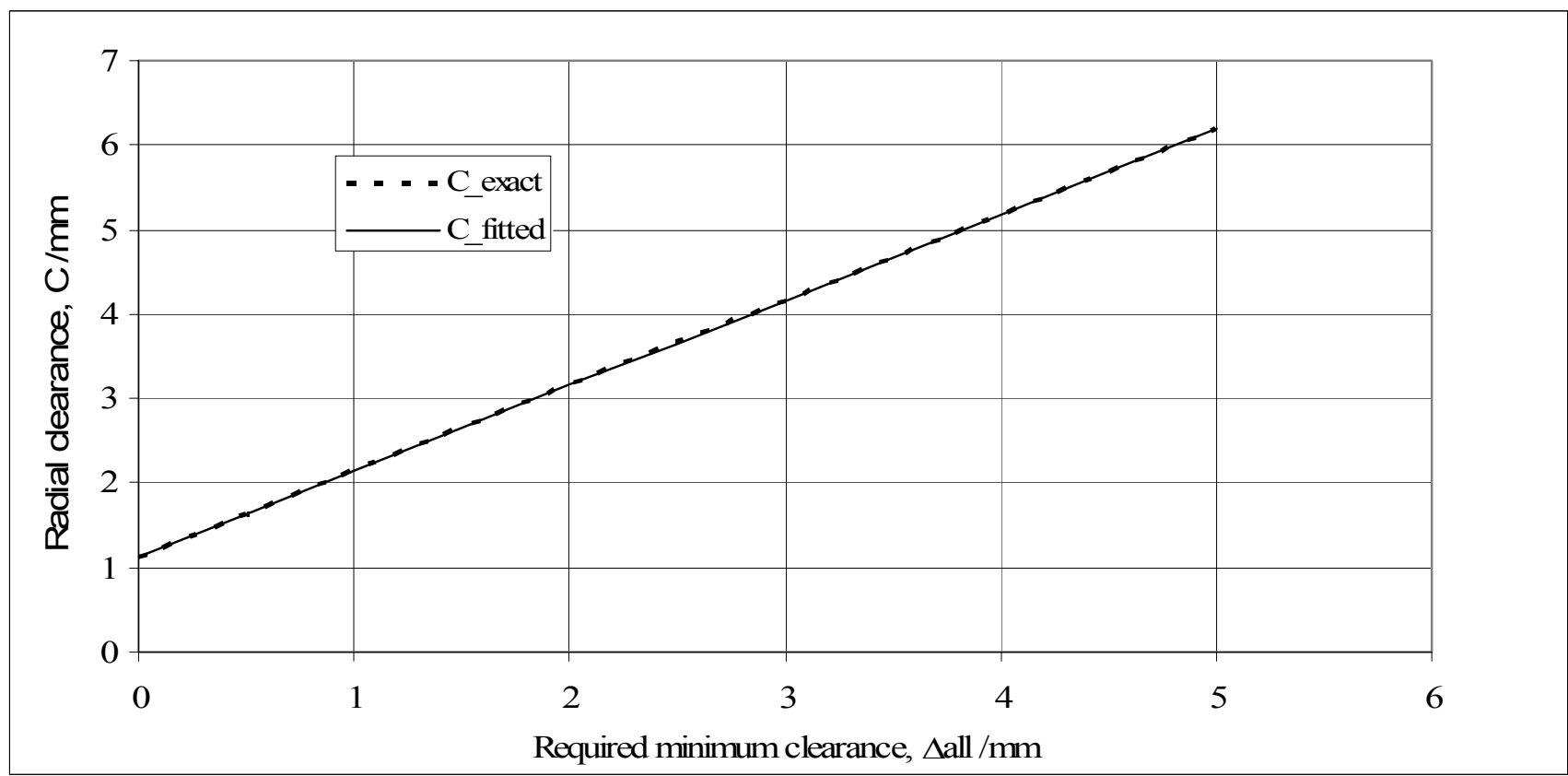

Figure 10. The $\mathrm{C}-\Delta_{\text {all }}$ linearity of the fitted model, for $\mathrm{b}=0.14$ and $\mathrm{L}=220 \mathrm{~mm}$. 